\title{
SOME INSPIRING CASES OF ACUTE RHEUMATISM
}

\author{
BY \\ F. JOHN POYNTON, M.D., F.R.C.P. \\ Physician to University College Hospital, and The Hospital for \\ Sick Children, Great Ormond Street, London.
}

The difficulties in finding and clearing any path by which the various morbid conditions included under the general term of 'rheumatism' can be either linked up to one another or definitely separated, present a great problem in practical medicine. For this reason it must be a cardinal principle to endeavour to make some definite base from which further explorations can be undertaken and to which return can be made for a fresh start, should these ventures prove unsuccessful. This is the essential reason for establishing a definite disease, the acute rheumatism of childhood, and I am of opinion that in so far as any disease is an entity this acute rheumatism is definite. True it may shade off in many directions but the mean of numberless observations is a morbid condition with a well established pathological anatomy and clearly recognised cardinal symptoms.

The study of such a disease may be directed to the clear exposition of its classical features, its course, prognosis and treatment; or again may be directed to the more unusual cases from the standpoint of their bearing upon the wider problem of rheumatism. Such cases I term 'inspiring' because they lead our thoughts out of the ordinary groove, and though these thoughts may be of the nature of speculations and therefore always open to error, they are nevertheless, as it were, mental tonics. Some of these unusual cases are commented upon in this article and the lines of thought they suggest indicated. I am, then, dealing with some unusual examples of, or contingent to, acute rheumatism, which are not only of interest in themselves, but are valuable because they lead one to think of the variable behaviour of the rheumatic infection or infections, and may possibly give to some investigator who is carrying on the exploration of rheumatism in general some hint which may lead to further advances. There is no doubt one danger to be avoided in a study of this kind, and this is to allow the unusual case to upset our standard. For example, a case will be touched upon in which the prominent feature was the occurrence of many nodules without any grave disease. Dr. Cheadle long ago pointed out that the occurrence of nodules meant severe heart disease and not rarely a fatal illness. Statistics have entirely supported this view, and $m y$ own figures from various series have convinced me of its essential truth. This unusual case cannot then be used to controvert a general rule, but it can be used to stimulate interest in the occurrence and meaning of these nodules.

In another example the commencement of an attack of acute rheumatism with acute heart block as the first manifestation leads one to think of the rheumatic myocardial lesions, always a difficult yet most important aspect of rheumatic heart disease, 
In a third case the occurrence of an enlargement of the thyroid gland coincident with a severe cardiac rheumatism reminds us that for many years acute rheumatism has been cited as an antecedent of exophthalmic goitre, and yet in a wide experience, it is only very occasionally that I have been able to trace a direct connection between acute rheumatism and an obvious disturbance of the thyroid, though I have with comparative frequency found a record of previous acute rheumatism in the history of exophthalmic goitre.

CASE 1. Acute rheumatism starting with heart-block. The first case was that of a boy, W. S., aged 13 years, a nervous but athletic lad who on January 17th, 1923, developed a sore throat, not of great severity but definite. Four days later he suddenly had pain in the chest, fainted and vomited, and these fainting attacks were repeated. A natural suspicion was diphtheria. There was, however, not the least evidence of this infection, but the condition was one of obvious heart block, the auricles beating at 72 , the ventricles at 36 . I considered the symptoms to be of the Stokes-Adams type, but the cause of the heart-block mysterious. The mystery was, however, soon cleared up, for shortly afterwards he developed chorea and acute multiple arthritis with endocarditis of the mitral and aortic valves. His condition became a very serious one and the illness extended over months but the heart block was transitory and lasted only a few days. A slight mitral systolic bruit with cardiac dilatation was detected after this phase of heart block had passed and the boy kept strictly in bed and it was then that the chorea and the acute arthritis showed the cause of the heart block. The chorea was an inter. esting one. It began quietly but eventually developed great severity and there were complete dumbness and urgent illness on account of the heart disease. He made eventually a good recovery but the heart was severely damaged by the endocarditis of the two valves.

This onset of acute rheumatism with heart block has been repeatedly observed, and we owe to Dr. G. A. Sutherland his experience of the-value of adrenalin subcutaneously in 1-1,000 solution for dealing with the urgent symptoms. To me it is also an event of extreme interest, for so far back as 1899 I demonstrated at the then Royal Medico-chirurgical Society the scattered focal lesions produced by acute rheumatism in the myocardium. All know them now as Aschoff's nodules and as having a special structure. When the work of the late Sir J. Mackenzie, Sir T. Lewis and all that great band of investigators threw light upon arrhythmias, it was obvious to me that the scattered lesions of acute rheumatic carditis might some of them damage the conducting system. Clinical experience, as shown by these cases of heart block gives support to that pathological work of the last century, though perhaps I am surprised that the phenomenon of acute heart block is not more frequent in the carditis of childhood. Of more general importance is the history of this case as an illustration of my central theme, the difficulty of the study of rheumatism and the need for inspiration. This boy, save for the heart block and the definite sore throat, had no signs of acute rheumatism, yet he developed an attack of a prolonged and severe nature, leaving permanent cardiac damage. In these days, very rightly, great attention is being drawn to the prevention of the disease in the young, but it is obvious that if such a severe case as this can begin so mysteriously, we must be prepared to be very patient while a greater knowledge of the disease is being built up and expect many difficulties in diagnosis even in this one branch of the general study of rheumatism, the acute disease in childhood. When we think of rheumatism in general we 
should indeed be short-sighted if we did not forsee how long and intricate the study of such a problem will be, and how far we must travel from terms such as 'Intestinal toxæmia,' ' radio-active waters,' disturbance of 'endocrine secretion' and so forth. I do not say these terms are valueless, far from it ; but they are at present only indications for research.

CASE 2. Multiple nodules with apparently normal heart The second case was a female child, P. B., aged four years, who had complained for a month of pain in all the joints and screamed when they were touched. There was also a history of abdominal pains which had been complained of for about 12 months and which were most definite before meals. At times also a rash appeared coincident with an outbreak of the pain and when this rash was severe blisters formed. There had been no previous attack or history of rheumatism in the family. On admission on October 16th, 1925, though rather pale she did not seem ill, and her appetite was good. There was complaint of pain in the elbow joints but no swelling and numerous subcutaneous nodules were seen along the extensors of the metacarpo-phalangeal joints of the hands over the left fibula above the left external malleolus and also over the back of the head.

The appearance of the hands, owing to the nodules, superficially resembled that of an osteo. arthritis in an elderly person. There was slight creaking on movements of the knee joints. For the pain in the abdomen no explanation was forthcoming unless it was neuro-muscular. $\mathrm{Her}$ heart was normal as judged by repeated clinical examinations. There was no chorea. The throat appeared to be healthy and there was no history of tonsillar trouble.

In brief, here was a child whose striking clinical signs were subcutaneous nodules of the classical type, and yet the heart was not affected and the child not apparently seriously ill.

We kept her in the hospital for over four months to make a careful study of her case and effect of the treatment. Throughout all these weeks her temperature was normal but crop after crop of nodules appeared on hands, elbows, knees, ankles and occiput; from time to time, also, a multiform erythema developed. chiefly on the abdomen, and the abdominal creases showed a peculiar redness. One nodule was removed for investigation, but the culture after four days was sterile.

The heart was occasionally quickened to 100 per minute, but averaged about 80 . A sinus arrhythmia was frequently detected, but never a murmur.

General treatment was tried with salicylates, tolysin, and lastly anti-rheumatic vaccine; also radiant heat and massage and, later, artificial sunlight were employed.

She left the hospital on March 12th, 1926, by request, better than when she came in but not really free from rheumatism.

If we take the nodules as the index for the result of the treatment, in my opinion, not one of the remedies influenced the case.

Five days after leaving hospital she was brought back with slight but definita chorea, and it may be added here that her parents were both highly nervous people. Her heart was now quickened and the pulse 120 per minute and there was some dilatation, the impulse being one inch outside the left vertical nipple line. The first sound was short ; there was sinus arrhythmia but no murmur or thrill. Once again there was a multiform erythema over the abdomen and an entire absence of fever. The chorea was at first slight and rapidly subsided and the heart became regular; but more nodules commenced to appear and a commencing mitral stenosis was suspected on account of an abrupt first sound. Next, the chorea now commenced to imitate the behaviour of the nodules by recurring and this time the child became quite dumb and the limbs showed the extreme paralytic type of the affection. The mentality also became very dull and the child looked seriously ill. There was a slight rise in the temperature for two days and then it returned to the normal once more.

It is very remarkable that in three cases in this article there wasdumbness with the chorea. Well known as this symptom is, in my experience it is uncommon. Inability to speak on account of inco-ordination of the tongue 
and lips is frequent enough, but this absolute speechlessness with no effort to attempt articulation is exceptional and a most striking clinical feature. This condition may last for weeks, but I believe myself correct in saying recovery is always complete. All these three children were excitable and intelligent even for rheumatic children, and each one it will be noticed had a very unusual form of acute rheumatism.

It may be asked, why call a case with no fever 'acute rheumatism' and the answer is that the most terrible cases of pericarditis in children are those which run a course with no fever. After the Great War two such cases were under my care, reacting to no remedies and dying in a few weeks without the slightest reaction. No illness could have been more striking in its deadly virulence.

I have seen this child recently: she is much improved and has no cardiac lesion, and the nodules have also disappeared.

The occurrence of subcutaneous nodules as the prominent feature in an attack of acute rheumatism is a fact that has been known for a long time, but such cases are most unusual, and on no account should they be allowed to alter the general rule that it is the severe cardiac cases in which these lesions are usually found. Every one who collects a series of cases of acute rheumatic carditis and analyses it, will find among the fatal ones a high incidence of subcutaneous nodules. For my own part, I have long been hammering at a closed door in trying to understand what is the real meaning of these nodules. It would appear now that the type of lesion is a response to streptococcal infection and I look upon them as local infections and am aware from an investigation I made with my colleague Dr. G. F. Still, that changes occur in the subcutaneous tissues in proximity to nodules which are identical but differ in that they do not form nodular projections because they are not over prominences. Dr. V. Coates has recently directed our attention to a miliary type of these nodules and once more roused our interest in them. Does, for example, the subcutaneous infection arise from the cardiac valves; I do not mean from gross emboli but from scattering of the infective agent ? These observations seem to throw a little light upon the problem. Dr. Paine and I found and grew the diplococcus from them and actually saw it in a dilated capillary of one of them, and others have succeeded in demonstrating them. Why do they vary in frequency in different years? Again, what influence has movement and pressure upon their formation?

Undoubtedly we can see there is more to be learned about acute rheumatism before we can thoroughly interpret its various clinical features. In the Archives of Disease in Childhood, August, 1926 (pp. 183-193) a valuable paper was published upon the subject of rheumatic nodules by Vincent Coates and Carey Coombs.

CASE 3. Severe rheumatism with thrombosis of inf. vena cava. The patient, a boy, F. W., aged 13 years, was admitted to University College Hospital, on October 10th, 1922. Six months before this he had been noticed to have had twitching movements of the face and hands for about a fortnight. Three weeks before admission, he had an attack of acute rheumatic arthritis affecting the knces, elbows and hands, which subsided in a week, but was followed by chorea 
which was at first mild but of the paralytic type. On admission, he was dumb and there was cardiac dilatation with mitral endocarditis. Four days later there was arthritis of the left elbow. The chorea was very unusual in type, with incontinence of urine, refusal to obey any simple commands and a mental state bordering closely on dementia varied, at intervals, by emotional outbursts. Later there were stertorous breathing and nystagmus and the pupils were widely dilated. The cerebrospinal fluid was sterile on culture. I would add here that in the last 18 months I have seen two cases of chorea with hyperpyrexia. Both of them recovered. To return : on October 26, the right leg commenced to swell from ankle to thigh. This swelling, which was an odema, increased and the veins of the abdomen below the umbilicus became enlarged. On November 6 the left ankle became œedematous and the clinical picture of a thrombosis at the lower end of the inferior vena cava developed. During this period of urgent illness there were also pleurisy, grave suspicion of acute pericarditis, considerable but not unusual fever and multiple arthritis. Eventually he made a good recovery and proved to be a most intelligent boy. A slight degree of mitral stenosis was present and though the cedema of the legs mostly disappeared in bed it returned in the erect position. He was taken in again later for observation. His condition then resembled precisely one which is sometimes seen with thrombosis of the inferior vena cava after typhoid fever. Large veins were visible over the abdomen below the umbilicus in which the blood stream coursed upward. There was slight œdema of the legs when at rest and considerable œedema after standing and walking. The right leg was more swollen than the left. The general condition was otherwise good. All later efforts to trace this case have failed.

In 1898 I described three cases of extensive various thrombosis in acute rheumatism all affecting the upper extremities; in one child the superior vena cava and all its main tributaries were full of clot. For years I did not meet with another case, but some four years ago I saw three more all implicating the upper extremities and all associated with severe and active heart disease. This is the only case in which I have seen the lower extremities affected. In my early cases $I$ attempted to discover in a fatal case whether it was a primary phlebitis, and to this view I inclined, or a primary thrombosis, but none of the other cases $I$ have seen since was fatal. The other interesting point in this case was the intense mental change. On the whole, great mental degradation is rare in chorea, but cases in childhood occur, as I have seen, in which hallucinations have been prominent and the chorea otherwise not severe. In the acute rheumatism of adults there may be acute insanity without chorea, though this must not be mistaken for the delirium from salicylate poisoning or that which is sometimes met with in an attack of acute pericarditis. The two acute cases of insanity under my personal observations both made good recoveries.

CaSe 4. Urticaria and painful joints. The next unusual case, which I cannot claim as an acute rheumatism, was a boy, W. S., aged $8 \frac{1}{2}$ years, admitted to hospital December 19 , 1925, for swelling of the joints and a rash, and sent in by my colleague, Dr. D. Paterson, as probably a case of Still's disease. In September he was hop-picking in Kent, when the rash suddenly appeared over the face, trunk and limbs and a few days later swelling of the joints of the fingers and toes occurred. Since that date there had been repeated recurrences, and the wrists, elbows, neck and shoulders had been implicated. The rash caused much irritation when it appeared and sometimes accompanied and sometimes followed rapidly upon the arthritic swellings. His mother attributed the illness to eating a "toad-stool," but there had never been any vomiting or diarrhœa or nervous symptoms such as we associate with such poison. The family and personal history threw no light on the condition.

On admission he looked tired and ailing, but except for a pale pink macular rash upon the thighs, buttocks, forehead and at the roots of the hair, there were no clinical signs. 
A week after admission the temperature, previously normal, rose to $101^{\circ} \mathrm{F}$, and continued as an intermittent pyrexia for ten days and then, after twenty four hours quiescence,ran for weeks ${ }^{*}$ an irregular course, occasionally reaching up to $102^{\circ}$ or $103^{\circ} \mathrm{F}$., but usually ranging between $100^{\circ} \mathrm{F}$. and normal. His general condition was one of fatigue. Three days before the first rise of temperature, there was pain in the left ankle; two days later this swelled and then the finger joints swelled and were painful and an erythematous rash appeared over the trunk, urticarial in type. There was no enlargement of the spleen or lymphatic glands.

Dr. H. F. Turney, my house physician, investigated the protein reactions and found a definite and general sensitisation to all the proteins. Blood culture was negative. Culture from the urine was negative. Bacterial examination of the fæces showed no abnormality. Dr. A. H. M. Gray also kindly examined the case for me and confirmed the essentially urticarial character of the eruption, suggesting the presence of some local focus of infection. His tonsils were apparently normal and had never given trouble, but three unhealthy teeth were extracted. Every search for a definite focus was negative. Aspirin relieved the arthritic pains, and on account of the hypersensibility to protein, a course of intravenous peptone injections was given by Dr. Turney. Armour's No. $25 \%$ peptone was used and two minims of this were made up to ten with normal saline. The injections were given twice a week and the protein increased each time by 3 minims. Salicylates and Tolysin were also tried and careful dieting, but none of us felt that any of our treatment had influenced this mysterious illness which continued to show the same recrudescences, and supported Dr. Paterson's view that it was a case of the Still type, in spite of the absence of an enlarged spleen and lymphatic glands. Eventually he gradually recovered, yet quite recently he had some pain in his ankles.

This case, whatever may be its exact nature, appears to me of such interest from the fact that a definite sensitisation to protein was demonstrated. It throws, as it were, a glimmer of light on the metabolic problem in disorders of this type. We all recognise the striking example of metabolic disorder with arthritis in the well known disease gout, and the very existence of such a condition must always be a perpetual reminder of the possibility of some subtle change in metabolism in such conditions as acute rheumatism and rheumatoid arthritis and this is supported in acute rheumatism by the well known hereditary factor. In acute rheumatism there seems little doubt that the element of infection does in many cases in childhood dominate the illness, but the problems still remain as to whether, on the one hand, the predominance of the infection is not in part the result of some latent metabolic weakness, and on the other, whether in the prolonged and recurrent type of the disease the infection itself does not gradually produce such a weakness.

CASE 5. Malignant endocarditis supervening on rheumatic carditis. This boy, C. H., whom I watched carefully for ten years, interested me very much, for in 1915 he was under my care in hospital with acute rheumatism and severe endocarditis damaging the mitral and aortic valves. It is these cases where both valves are affected in which I suspect a malignant tendency, for the fact of the multiple lesions occurring in the two valves so closely situated to one another suggests in itself this type, although at first both these lesions may scar and quiet down.

In 1917 he had another attack of acute rheumatism and in 1918 a severe pericarditis with prolonged fever and extreme dilatation of the heart. His life hung long in the balance, but after many months in hospital he made a good recovery and came to report to me every six months or at any time he had warnings of rheumatism.

He was a very steady boy with good parents and became a clerk. Year after year he reported to me and grew to be a well developed young man in spite of his much damaged heart which showed free aortic regurgitation and mitral stenosis. All these years his health remained remarkably good and he kept at work, taking good care of himself. 
In May, 1925, he began to feel generally ill and came to hospital, but by a sad chance I did not visit that day and he unfortunately returned home without seeing me, intending to come the next week. In the meantime he developed what was called "influenza" and took to his bed with much pain over the heart and great illness.

He was finally admitted on June 5 extremely ill with high fever. An important point in his case was the condition of his teeth, which were unhealthy. One had been removed some weeks before and the gums had been septic. In the hospital cultures from the blood gave a pure growth of a non-hæmolytic streptococcus viridans.

It is not necessary to dwell upon the details of a very distressing and acute malignant endocarditis with cerebral and arterial embolisms and rigors ending in death, but a few details of the necropsy are of value.

There were exuberant vegetations on the mitral and aortic valves, numerous infarcts in the spleen, both recent and old; an embolus in the right common iliac artery with another in the left popliteal artery and a large embolus with consequent softening of the right parietal lobe. In spite of the high fever and repeated rigors there was no suppuration.

This case in turn opens up the well-known and most important question of progressive or malignant endocarditis which has rightly attracted great attention in the world of medicine. I need not dwell upon my own views on this question which have been so often expressed in various papers, but put forward some definite questions for consideration. The first is this. Is it advisable to make a time definition in these forms of endocarditis? By this I mean, is it advisable to think of a disease in the terms of acute, subacute and chronic infective endocarditis? May not these be phases of any infective endocarditis ? Secondly, does not such a terminology suggest a non-infective endocarditis, and are we prepared to admit such a condition ? Thirdly, though rheumatic endocarditis generally heals as do the nodules, the chorea and arthritis, are we prepared to say it always heals, and if it does not, what kind of illness do we think will result ?

Attempts to answer these questions at once lead us into the midst of some of the greatest problems in the general study of rheumatism.

CASE 6. Chorea and enlargement of thyroid. The last case was that of a girl aged 16 who at the age of 11 years had a short attack of chorea and in September, 1924, scarlet fever followed by rheumatism. In 1925 she was operated upon for enucleation of the tonsils and removal of adenoids, after which she had no more rheumatic pains, but during this last year, except for an interval in the summer, suffered from a moderate and stubborn chorea. This girl on admission to hospital. November 28, 1925, had definite chorea and aortic endocarditis, but the most interesting feature was an enlargement of the thyroid gland moderate in degree but very definite. It was firm in consistence and the right lobe was rather larger than the left. Arterial pulsation could be detected and there was fine tremor of the fingers, but no exophthalmos, and the pulse rate which ranged around 80 to 90 per minute was no swifter than would be expected from the organic heart disease. This thyroid swelling had been noted for some years but could not be accurately dated and varied in degree, and increased with exacerbation of the chorea.

In hospital she did well and the thyroid diminished in size. Further observation and treatment by Lugol's solution of iodine confirmed the general improvement and the consistence of the thyroid became firmer and the fine tremor diminished. She is still under observation.

Once again this case opens up a field for thought and observation and Dr. L. J. Llewellyn has in particular laid stress upon disorder of the thyroid in rheumatism. 
By a coincidence while engaged upon this article another case of acute articular and cardiac rheumatism in a female patient followed at once by enlargement of the thyroid and the definite signs of Graves's disease came under observation. In her case there had been the factor of great nervous strain preceding the attack of acute rheumatism.

At the present time also I have under my care a third case, a child aged 13 years, a girl of very excitable temperament, who had been under my observation in hospital for chorea in 1923, and again with a very severe attack in 1925. On the second occasion her eyes appeared to be more prominent but there was no enlargement of the thyroid gland. For the third time she was admitted on December 18th, 1926, for a recurrence of chorea of a fortnight's duration. She now showed an enlargement of the thyroid affecting both lobes, obvious exophthalmos, Stellwag's sign, fine tremor, and a rapid heart with an occasional systolic murmur in the mitral area. It would appear that in 1925 there was some hyperthyroidism as shown by the commencing exophthalmos, but that the thyroid was not enlarged or, to be more cautious, not sufficiently enlarged to attract attention. During the interval between 1925 and 1926 the child was apparently well and her mother had not noticed any swelling of the neck, but when admitted to hospital with the renewed chorea, the enlargement was very apparent and there were also other clear signs of exophthalmic goitre.

It is no new idea that the thyroid is affected in acute rheumatism, but when one looks back upon the many cases of the disease one has seen, the firm impression is left that if the thyroid is often affected the gland itself rarely shows any gross evidence of disease. In some cases of chorea I have noticed enlargement of the thyroid, but in hundreds $I$ have seen no change. Yet we must allow that there may be a subtle alteration in the secretion without any clinical evidence, and possibly prolonged studies of the basal metabolism may prove a source of further light. Acute rheumatism is a disease in which we might well expect some thyroid change, when we recall the peculiar nervousness of the rheumatic and also those cases of irritable heart in the rheumatic in which we are doubtful whether the condition is due to some neuro-muscular damage to that organ, or to some circulating poison or disordered metabolism which is disturbing a heart but producing no definite myocardial lesion. The same possibilities, it will be remembered, have also arisen over rheumatoid arthritis. No thoughtful observer who accepts acute rheumatism as infective in origin can doubt that in this disease there is also probably some peculiar metabolism, whether innate or produced by the poisons linking themselves into the very tissue cells of the patient. This is an aspect of rheumatism which has always attracted me because of the inveterate course of many cases and the different behaviour of the lesions at different ages.

It may be thought that I have wandered far afield into speculation, but this is the result of inspiring cases. Such do not allow us to be content with bare enumeration of symptoms and signs. There are occasions when it is advisable to keep on the beaten track and drive home the essential features 
of classical cases, but equally there are occasions when it is useful to imagine what great fields there are before future investigators. It is sometimes said that all the easy things in medicine have been already done, but these easy things were not easy when they were still problems, and future investigators have, among other encouragements, the rapidly developing science of biochemistry. Looking forward one can see that some day that great gulf will be slowly bridged which will enable the physician to link together the bacterial poisons on the one hand and the metabolic disorders produced by them in the human tissues on the other. This is one of the great difficulties in the study of rheumatism. No one is a firmer supporter of the belief of infection as a factor than I am, and I venture to think no one is less convinced that mere infection is the sole explanation of these diseases. But at the moment we are working in an atmosphere of mist because we do not know sufficient of the bio-chemistry of these diseases to recognise the bacterial and human sides in the many divergencies from the normal which are presented. 